\title{
Articaine vs Lidocaine: A review
}

\author{
${ }^{1}$ Dr Syed Gufaran Ali, ${ }^{2}$ Dr Sanjyot Mulay, \\ ${ }^{I}$ PhD Scholar, Faculty of Dental Sciences, Pacific Academy of Higher Education \& Research University, \\ Udaipur-313024 \\ ${ }^{2}$ Professor, PG \& PhD Guide, Department of Conservative Dentistry \& Endodontics, Dr. D Y Patil Dental \\ College \& Hospital, Pune-411018
}

\begin{abstract}
The introduction of local anesthetics revolutionized the practice of dentistry. Prior to their introduction, general anesthesia was the only viable method of managing surgical pain. Among various local anesthetics available in India, lidocaine is most widely used. But lidocaine cannot provide 100\% anesthesia, this leads to development of other anesthetics, one such is articaine. However, little to no evidence-based medicine exists demonstrating any superiority of articaine over other available local anesthetics. However dentists in clinical practice have claimed that articaine possesses properties that other local anesthetics don't.
\end{abstract}

Keywords: anesthesia, articaine, lidocaine.

\section{Introduction:}

Local anesthetics form the backbone of pain control techniques in dentistry. ${ }^{1}$ Effective local anesthesia is a must in the management of painful endodontic emergency. Success rate of anesthesia may decrease drastically in patients with irreversible pulpitis, because of:

a) Local acidosis caused by tissue inflammation.

b) Activation of nociceptors by inflammatory mediators.

A range of local anesthetic drugs have been used in dentistry, amongst which lidocaine is the most popular. Articaine, a safe local anesthetic introduced in April 2000 in the United States, has a reputation of providing an improved local anesthesia. Since its introduction in Germany in the early 1970s, articaine has been compared in double-blinded, randomized, controlled clinical trials to each of the other available local anesthetics. To date, only few clinical trials have demonstrated any superiority of articaine as compared to lidocaine. Endodontists have become enamoured with the drug as a more definitive means of achieving profound anesthesia to permit painless pulpal extirpation in "hot" mandibular molars - the most difficult teeth to anesthetize successfully, yet again in the absence of any published clinical trials demonstrating this advantage.

\section{The basic properties of articaine and lidocaine:}

The chemistry and pharmacology of a local anesthetic can give valuable information about which clinical effects you can expect when you use them. The most important ones for both articaine and lidocaine are listed in table 1.

Table 1: Comparison of articaine and lidocaine -

\begin{tabular}{|c|c|c|}
\hline Substance & Articaine & Lidocaine \\
\hline Chemical formula & $\begin{array}{l}\text { 3-N-Propylamino-proprionylamino-2-carbomethoxy-4- } \\
\text { methylthiophene hydrochloride }\end{array}$ & $\begin{array}{l}\text { 2- Diethylamino 2, 6-acetoxylidide } \\
\text { Hydrochloride }\end{array}$ \\
\hline Classification & Amide & Amide \\
\hline Molecular weight $^{2}$ & 284.38 & 234.34 \\
\hline
\end{tabular}




\section{1) Concentration of anesthetic solution:}

\section{Comparison of articaine versus lidocaine:}

Articaine is delivered as a $4 \%$ solution in as opposed to lidocaine which is $2 \%$. It may be speculated that if there is a toxic local metabolite involved, it may manifest toxicity simply due to the higher concentration. This also means that when the same recommendations for the maximum doses are applied, one can inject twice as many carpules of lidocaine when compared with articaine. This is important to be aware of during situations where more anesthetics have to be re-injected. But, there is another feature that has to be taken into account when one is re-injecting anesthetics and is concerned about the maximum doses, and that is the elimination halflife. As mentioned before, articaine contains an additional ester group that is quickly hydrolyzed by plasma esterases. ${ }^{6}$ This gives articaine an elimination half-life of approximately 20 minutes, compared to that for lidocaine which is approximately 90 minutes. This makes re-injection of articaine safer, since the majority of that initial dose are metabolized after approximately half an hour, and the re-injected dose will not be added to the initial one. ${ }^{7}$

\section{2) Lipid solubility:}

Lipid solubility affects the anesthetic potency. Increased lipid solubility permits the anesthetic to penetrate the nerve membrane, which itself is $90 \%$ lipid, more easily. ${ }^{3}$ Articaine differs from all other amide local anesthetics, in that it is derived from thiophene. As a result, the articaine molecule does not contain a benzene ring like the others but instead contains a thiophene ring. This renders the molecule more lipid soluble and therefore better able to cross lipid barriers, for example the nerve membrane. ${ }^{7}$

\section{3) Protein binding:}

Protein binding affects the duration. Increased protein binding allows anesthetic cations to be more firmly attached to proteins located at receptor sites. Thus the duration of action is increased. Articaine has high protein binding.

\section{4) Metabolism (Biotransformation):}

Metabolism of local anesthetics is important, because the overall toxicity of a drug depends on a balance between its rate of absorption into the bloodstream at the site of injection and its rate of removal from the blood. Approximately $70 \%$ of the dose of injected lidocaine undergoes biotransformation in patients with normal liver function. Significant liver dysfunction or heart failure represents a relative contraindication to the administration of amide local anesthetics. ${ }^{3}$ Articaine differs from other amide local anesthetics, in that it has an extra ester linkage (COOCH3). 90-95\% is metabolized in the blood, and only $5-10 \%$ in the liver. The major metabolic product of articaine is articainic acid. It is inactive as a local anesthetic, and systemic toxicity has not been observed. ${ }^{6}$ This finding is important because an active metabolite may affect toxicity and may exert undesirable side effects. In comparison, lidocaine has active metabolites. It is metabolized in the liver by the microsomal P450 enzyme system to monoethylglyceine and xylidide; xylidide is a local anesthetic and potentially toxic. $^{3}$

\section{5) Excretion:}

The kidneys are the primary excretory organ for both the local anesthetic and its metabolites. A percentage of a given dose of local anesthetic drug will be excreted unchanged in the urine, and this varies according to the drug. Articaine is largely excreted in the urine as the metabolite articaninic acid $(64.2+/-14.4$ $\%)$, followed by articainic glucuronide $(13.4+/-5.0 \%)$ and the parent drug $(1.45+/-0.77 \%) .{ }^{8}$ For lidocaine the excretion is also via the kidneys; less than $10 \%$ unchanged, more than $80 \%$ various metabolites. $^{3}$

\section{6) Pharmacodynamics:}

The potency of articaine is 1.5 times that of lidocaine and 1.9 times that of procaine, and the toxicity is similar to that of lidocaine and procaine. ${ }^{3}$ The unintentional intravascular injection can cause severe CNS and CVS toxicity. ${ }^{6}$

\section{7) Safety:}

Lidocaine, which is a relatively old local anesthetic, has been studied thoroughly for many years, and has well documented by various studies for their effects and side effects. The safety has also been under substantial investigation for many years. Articaine is not as old as lidocaine, although it was synthesized back in 1969. It has been used in several European countries for almost 30 years, and its safety has been well documented by several different studies. 
The unintentional intravascular injection of local anesthetic agents in dentistry can occur because of high vascularisation in this area. The risk of such an intravascular injection is up to $20 \%$ in conduction anesthesia of the mandibular nerve. ${ }^{8}$ The signs and symptoms of local anesthetic agent toxicity are referable to the CNS and cardiovascular system. The initial subjective signs of CNS intoxication are general feeling of light headedness, dizziness, disorientation, drowsiness, anxiety, excitement and visual and auditory disturbances. Objective signs of early CNS toxicity consist of shivering, muscular twitching and tremors of the facial muscles. Oertel et al. (1997) showed that signs of CNS toxicity after intravascular injection of lidocaine were observed more frequently and at a higher degree of severity when compared with articaine. The cardiovascular parameters did not change. ${ }^{6}$

The immunogenic potential of articaine is very low. Allergic-type reactions that have been reported with articaine include oedema, urticaria, erythema and anaphylactic shock, and the frequency is comparable with that of lidocaine. ${ }^{9}$ There are however several factors that may alter the predictability of allergic drug reactions. Age, genetics, frequency and duration of drug administration and route of administration are all factors that may contribute to the predictability. ${ }^{10}$ Therefore patients allergic to articaine likely would be allergic to lidocaine and other amide local anesthetics.

Some authors claims that articaine like prilocaine is capable of producing methemoglobinemia. But when used as directed for dental anesthesia, the occurrence of this side effect is highly unlikely, and no cases has been reported following the use of recommended dosages for dental local anesthesia. ${ }^{7}$ Earlier formulations of articaine and other local anesthetics contained a bacteriostatic, antifungal and antioxidant preservative for the local anesthetic itself, called methylparaben. It was proved allergenic, and was not removed from all formulations of articaine until the mid 1990 `s. This is one of the reasons that approval for the use of articaine was not sought in the United States until 1995.

\section{8) Efficacy:}

An important property to look at when it comes to efficacy is the lipid solubility expressed by the partition coefficient. The lipid solubility determines to what degree the molecules penetrate nerve membranes. Several studies have been conducted to find out what the properties are for articaine, and the results of these studies vary. Oertel et al. (1977) reported a partition coefficient for articaine of 52, similar to that of lidocaine (N-octanol /buffer, $37{ }^{\circ} \mathrm{C}$ ). ${ }^{11}$ Another property which is important for the diffusion is the molecular configuration. Articaine contains a thiophene ring instead of benzene like lidocaine. This gives the molecule better diffusion properties compared with lidocaine.

Similar to other amide local anesthetics, articaine blocks sodium channels at a lower concentration than potassium channels, but lower concentrations of the thiophene derivate than of the benzene derivates are needed to block the ionic channels. Because of the higher partition coefficient of articaine, it seems that the action on these channels seems not to follow mere lipid solubility properties of the neutral drug. Differences in the interaction of local anesthetics with ionic channel proteins might in part be correlated to different binding properties to plasma proteins, as indicated by a higher affinity of articaine to plasma proteins as compared to lidocaine. ${ }^{12}$

\section{9) Use in paediatric dentistry:}

In order for a local anesthetic to become popular, it is important that it is useful in a wide range of situations. Lidocaine has been used for both adults and children for more than five decades, and paediatric dentistry is for sure an important area. Previously articaine was not be used in patients under 4 years of age, because safety and effectiveness in this group has not yet been seriously investigated. But now there are studies which shows that articaine is likely safe for children under 4 years of age. ${ }^{12,13}$ When used in paediatric dentistry, it is important to remember that articaine is in a $4 \%$ solution, and that maximum dose for children is the same as for adults; $7 \mathrm{mg} / \mathrm{kg}(0.175 \mathrm{ml} / \mathrm{kg})$. For simple procedures the recommendation is $0.04 \mathrm{ml} / \mathrm{kg}$, and for complicated procedures $0.07 \mathrm{ml} / \mathrm{kg}$. It is important to have in mind that when you administer local anesthetic to children with small weight, the maximum dose can easily be reached.

\section{0) Use in geriatric patients:}

Aging is associated with physiologic changes which could alter pharmacokinetics of drugs. Age-related changes in pharmacokinetics affect drug absorption, distribution metabolism, and elimination. Increase in body fat, decrease in lean body mass, and total body water, changes in hepatic metabolism, and renal elimination capacity in the elderly are of particular clinical significance. These changes should be taken into account when choosing drug therapy for older patients to minimize adverse effects and maximize potential benefits. Because the median age of the population is steadily increasing, more elderly patients are undergoing routine dental procedures for which local anesthesia could be required. ${ }^{14}$ 
Taken into account that articaine shows an age-independent metabolism, there should be no reason to change the dosage in elderly patients. But, it is important to remember that articaine is a highly serum protein bounded drug, and changes in binding to serum are also a factor that could affect pharmacokinetics in the elderly.

\section{1) Complications:}

A wide range of different complications can occur during or after the injection of local anesthesia. They can be divided into local complications, such as pain on injection, persistent anesthesia/paresthesia, trismus, hematoma, oedema and facial nerve paralysis, and systemic complications such as overdoses and allergic reactions.

Among all, paresthesia is the most common. Paresthesia can be defined as persistent anesthesia (anesthesia well beyond the expected duration), or altered sensation (tingling or itching) well beyond the expected duration of anesthesia. ${ }^{3}$ The definition of paresthesia also includes hyperesthesia and dysesthesia. Hyperesthesia is defined as increased sensitivity to noxious stimuli, and dysesthesia as painful sensation to nonnoxious stimuli. ${ }^{15}$ The symptoms are most commonly associated with mechanical trauma during surgical procedures. During the administration of anesthesia for a mandibular nerve block, the lingual or inferior alveolar neurovascular bundle can be traumatized by the sharp needle-tip, the movement of the needle, extraneural or intraneural hemorrhage from trauma to the blood vessels, or from neurotoxic effects of the local anesthetic.

A retrospective analysis of paresthesia after local anesthetic administration for nonsurgical dental procedures over a 20-year period, from 1973-1993, was done by Haas and Lennon in $1995 .^{16}$ The analysis revealed a higher-than-expected frequency of paresthesia with articaine, based on the number of cartridges used (2.27 per 1 million injections vs. an expected frequency of 1.20 per 1 million injections). There were no significant differences found with respect to patient age, patient gender, or needle gauge.

An interesting finding is the different frequency between paresthesia of the lingual nerve and the inferior alveolar nerve. The lingual nerve (tongue) is approximately twice as often involved as the inferior alveolar nerve (lip), 92 versus 42 cases. This finding is confirmed by Progrei and Thamby, which found the same distributes for the paresthesia (2000). ${ }^{17}$ The reason for this finding might be that in performing inferior alveolar nerve injections some practitioners change direction of the needle at the approximate depth of the lingual nerve. The sharp needle tip may lacerate the lingual nerve and/or artery on the initial or subsequent path. Another possible explanation might be that during a subsequent injection for the inferior alveolar nerve block, the needle might traumatize the more superficial lingual nerve but without the "electric shock" sensation, because the nerve is usually anesthetized on the initial attempt. ${ }^{15}$ The cause of the paresthesia may also be combination of neurotoxicity of the local anesthetic and trauma to the nerve. ${ }^{18}$

Another complication that might occur is paralysis of the oculomotor muscles, leading to diplopia and even temporary blindness. All such manifestations are transient and disappear on cessation of the anesthetic effects. In case of paralysis of the extrinsic musculature of the eye (especially the external rectus muscle), synchronic movement of the eyes becomes impossible, and diplopia appears. Such complications are possible when performing a posterior superior alveolar anesthesia. There are many different theories on the mechanisms behind these opthalmologic complications, and it is likely that the local anesthetic solution diffuses directly from the pterigomaxillary fossa, through the sphenomaxillary cavity, to the orbit. This would affect the ciliary ganglion, located between the optic nerve and the external rectus muscle of the eye. ${ }^{19}$ The symptoms develop immediately after the injection of the anesthetic solution and can persist for between 1 minute and several hours, though they only rarely exceed the duration of the anesthetic effect. Most of the reported cases in the literature are produced by lidocaine and mepivacaine, but they may also happen after injections of articaine. ${ }^{19}$

\section{Conclusion:}

One of the reasons why articaine instantly became so popular in many countries was due to its excellent efficacy. Dentists claimed that they seldom missed with the inferior alveolar nerve block, and that buccal infiltration in the maxillary arch often was enough before an extraction of a molar, because of articaine's bone penetration properties. This seemingly excellent efficacy is reported from many dentists from around the world, based on their daily clinical practice.

An important issue when comparing two substances as articaine and lidocaine are the methods used. In order to get statistical significant data, one needs a large enough material containing a sufficient number of subjects. Many studies fail to show differences because of this problem. This may be one of the reasons why articaine in different studies tends to be somewhat more effective than lidocaine, although not significant. 


\section{References}

[1]. Malamed SF. Local Anesthetics: Dentistry's Most Important Drugs, Clinical Update, CDA journal 2006:34;971 -976.

[2]. Jastak JT, Yagiela JA, Donaldson D. Local anesthesia of the oral cavity. 1st ed. Philadelphia, Saunders, 1995.

[3]. Malamed SF. Handbook of local anesthesia. 4th ed. St. Louis, Mosby; 1997.

[4]. Malamed SF, Gagnon S, Leblanc D. Efficacy of articaine: A new amide local anesthetic. J Am Dent Assoc 2000;131:635-642.

[5]. Oertel R, Richter K. Plasma protein binding of the local anaesthetic drug articaine and its metabolite articainic acid. Pharmazie 1998;53:646-647.

[6]. Oertel R, Rahn R, Kirch W. Clinical pharmacokinetics of articaine. Clin Pharmacokinet 1997;33:417-425.

[7]. Isen DA. Articaine: Pharmacology and clinical use of a recently approved local anesthetic. Dent Today 2000;19:72-77.

[8]. Bartlett SZ. Clinical observation on the effect of injections of local anesthetics preceded by aspiration. Oral Surg 1972;33:520-525.

[9]. Malamed S F, Gagnon S, Leblanc D. Articaine hydrochloride: a study of the safety of a new amide local anesthetic. J Am Dent Assoc 2001;132:177-184.

[10]. Maccoll S, Young ER. An allergic reaction following injection of local anesthetic: A case report. J Can Dent 1989;55:981-984

[11]. Borchard U, Drouin H. Carticaine: Action of the local anesthetic on myelinated nerve fibres. Eur J Pharmacol 1980;62:73-79.

[12]. Jacobs W, Ladwig B, Cichon P, Oertel R, Kirch W. Serum levels of articaine $2 \%$ and 4 \% in children. Anesth Prog 1995;42:113115 .

[13]. Wright GZ, Weinberger SJ, Friedman CS, Plotzke OB. The use of articaine local anesthesia in children under 4 years of age - A retrospective report. Anesth Prog 1989;36:268-271.

[14]. Oertel R, Ebert U, Rahn R, Kirch W. The effect of age on the pharmacokinetics of the local anesthetic drug articaine. Regional Anesth Pain Med 1999;24:524-528.

[15]. Dower JS. A review of paresthesia. Dent Today 2003;22:64-69

[16]. Haas DA, Lennon D. A 21 year retrospective study of reports of paresthesia following local anesthetic administration. J Can D ent Assoc 1995;61:319-330.

[17]. Progrei M A and Thamby S. Permanent nerve involvement resulting from inferior alveolar nerve blocks. J Am Dent Assoc 2000;131:901-907.

[18]. Kalichman MW, Moorhouse DF, Powell HC, Myers RR. Relative neural toxicity of local anesthetics. J Neuropathol 1993;52:234240 .

[19]. Penarrocha-Diago M., Sanchis-Bielsa J M. Ophtalmologic complications after intraoral local anesthesia with articaine. Oral Surg Oral Med Oral Patho 2000;90:21-23. 